\title{
Real-Time Safety Automobile Driver System
}

\author{
Azmi Shawkat Abdulbaki \\ College of Computer Sciences and Information \\ Technology University of Anbar, Iraq
}

\author{
Samir Abdulrasoul Khadim \\ University of Al-Ma'arif College Dept. of Computer \\ Science, Iraq
}

\begin{abstract}
The Driver Fatigue Detection (DFD) plays an important role in automobile safety and security. Driver fatigue is one of the leading causes of traffic accidents. Long-vehicles driving are needed to keep drivers under monitoring due to fatigue and more accidents. This monitoring include : eyes blinking, face expressions. Without these monitoring, the accidents is increasing and caused the driver death. Driver's tiredness and drowsiness are the major causes of traffic accidents on road. This proposed prototype is monitor the driver's fatigue level and analyze fatigue reason's (tiredness, drowse or a drunken ) and real-time DFD online. The proposed model depend on eye gestures (eyes blinking) and sensors such as an infrared camera (camera remotely) to detect driver expression. These sensors directly pointed towards the driver's face. This technology is not interactive with an outside driving situation .The system consist of Mesh network equipped Zigbee protocol, sensors and Xbee tag. The system analyze the variation of driver's eyes movement rate. According that parameters, the system can specify the level of driver's fatigue based on the response signals and alert driver. Practically. This system is robust, reliable, and accurate to detect fatigue levels.
\end{abstract}

\section{Keywords}

Driver Fatigue Detection, Eyes Blinking, E-Safety.

\section{INTRODUCTION}

According the World Health Organization (WHO) reports in 2001 ,there are more than 20,000,000 people killing with road accidents yearly in all countries of the world. The daily traffic accidents has become a tragedy of modern life [1]. Road accidents are the primary cause of death in the world. Mainly ,There are three types of techniques that are used for driver's vigilance consideration. The first two considerations are monitor the driver directly, whereas the third are monitor driver indirectly, described below[2]: (1) Driver's intellect status detection-physiological measurements-(tested by the medical instruments) (2) Install a private camera (visual cues) for driver's image continually in front of driver's face (3) The driving State(driving performance) such as(speed, braking and turning wheel). Physiological measurements are measure features of the driver as brain waves, eye movements and heart rate. Visual cues monitor fatigue indicator and serve the driver directly by installing the camera in front of driver's face. With visual cues, most of the fatigue-related information can be obtained from the driver's eyes [3].

The proposed system depend on monitoring driver's eyes , and count how many driver's eyes are closed in the second. By these monitoring, the indications of driver fatigue can be detected early enough to avoid a vehicles accident. In the fatigued-state, the eye blinks are decreased but in the normal rate( non-fatigued state), the eye blinks are increased. Driver's fatigue can be detected by variations in the steering wheel angle, vehicle position and vehicle speed [4]. The wakecondition is keeping the vehicle within the driving lane whereas a tiredness driver will make large changes in lateral position in order to keeping the vehicle within the driving lane [5]. The developed systems that monitors the driver's fatigue levels and alert the drivers to prevent accidents are very activity systems to keeping drivers in safe. The visual cues detect eye movement and facial expression. Visual cues that distinguish levels of the alertness of person are extracted immediately to detect the fatigue level of the driver [6]. This technology is active for detecting drowsiness by monitoring the response of the driver. This involves periodically requesting the driver to send a response to the system to indicate alertness. By monitoring the eyes of the driver, the symptoms of driver fatigue can be detected early enough to avoid a vehicle accidents[7].

\section{MOTIVATION}

The motivation of this system to make drivers, goods and vehicles in safe area through development of electronic control system (E-Control System). This technology presents real-time non-intrusive fatigue monitoring system which exploits the driver's facial expression (fatigue, sickness and drunken) to detect and alert fatigued drivers[7]. This technology measure all changes in physiological signals, such as brain waves, heart rate, and eye movements, and, measure all physical changes such as flabby posture, leaning of the driver's head and the open/closed states of the eyes[8]. The technology helps the two sides of trading (salesman and buyer) and the driver in safe and secure side additionally the goods especially expensive and important [9] .

\section{RELATED WORKS}

Many efforts on developing active real-time image-based fatigue monitoring systems have been reported in the literature. These efforts are primarily focused on detecting driver fatigue from facial expression or line of sight or eyelid movement or physiological signals. K.Subhashini Spurjeonl and Yogesh Bahindwar are proposed paper describes a realtime dedicated system for detecting driver-fatigue and drowsiness by break traditional way of drowsiness detection to make it immediately ; it utilizes face detection and eye detection to initialize the location of driver's eyes; after that an object tracking method is used to keep track of the eyes [10]. Vatanavongs Ratanavaraha and Duangdao Watthanaklang present a study the analysis of bus hazardous locations via data gathering using traffic accident statistics on National Highways according to results of black spot analysis on Thailand National Highways, the inspection using the accident frequency method ranked 30 accident risk spots from high to low accident frequency. Suggestions on short-term and long-term solutions were provided by this study[11]. Aryuanto and F. Yudi Limpraptono present paper a visionbased system for monitoring driver fatigue by divide the system into three stages: face detection, eye detection, and fatigue detection. Face detection based on the skin color segmentation. The skin color segmentation based on a normalized RGB chromaticity. After face is localized, eye is detected by projection technique of the gradient image. Then percentage of eye closure over time) is calculated and used to detect a fatigue condition[12]. Reinier Coetzer present a paper 
discusses the work that have been done to develop a robust eye tracker, which will ultimately be used to detect fatigue by monitor the driver by means of a camera that is installed in the vehicle to track the driver's eyes[13]. Qiang Jil and Xiaojie Yangthis was present a paper describes a real-time prototype computer vision system for monitoring driver vigilance. The visual behaviors include eyelid movement, face orientation, and gaze movement (pupil movement). The system was tested in a simulating environment with subjects of different ethnic backgrounds, different genders, ages, with/without glasses, and under different illumination conditions, and it was found very robust, reliable and accurate [14].

\section{DATA SET}

Data sets are collected from subjects showing spontaneous facial expressions during the state of fatigue i.e. using blinking of eye movements. We analyze the degree to which individual facial action units can predict the difference between alert and drowsy or moderately drowsy and acutely drowsy. How to extract the relevant feature set of filters for a person independent drowsiness detector is studied. The propose system uses sensor network for detecting the Driver's Fatigue.

\section{PRE-PROCESSING}

Initially, the system start pre-processing by capturing frame to normalize the illumination and reduce image noise before face and facial detection[15]. Next, the capturing image convert from RGB image to grascale image in order to analyzing the pixel intensity values in the converted image. Image enhancement performed to obtain denoising image using $3 \times 3$ median filter. Histogram equalizer can be carry out to minimize the various illumination parameters[9][16].

\section{EYE AND FACE FEATURE EXTRACTION}

In this technology, the concept of interest specific regions such as eyes and face was adopted to avoid some errors occurs during the computational. After specify the interest regions , the pre-processing occurs only on the specific places such as eyes and faces. To improve the facility of our study area (eyes), we must be moved eyes within the correctly detected frame[16]. The locations of eye's frame are retained in order to use with another eyes locations. This technique is very useful when the eye of the driver are closed or drowse Drowsiness often leads to an increase in the number of closed frames, mainly because the individual tends to take a longer time to re-open his eyes. In the present eye frame, the elapsed time from the last eye closure is measured when every time of both eyes detected closed[17].

\section{FATIGUE DETECTION TECHNIQUE}

The process of drowsiness during driving can be characterized by decrease the wakefulness. This state occurs because of work hard, fatigue or sick. drowsiness detection system used to detect drowsiness early .The system based on these factors[18]: (1) Driver State and Performance (2) Analyze blinking image sequences .

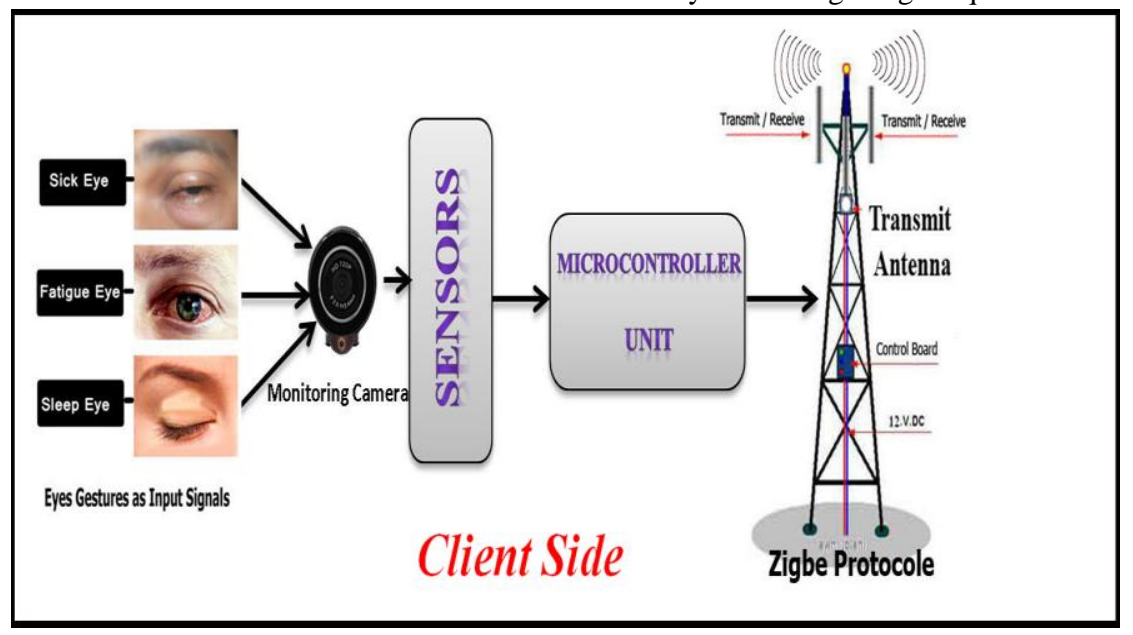

Fig (1) Driver's Section based on Xbee Tag

Additionally, fig.(2) shows the construct of the monitoring system in the server section . 


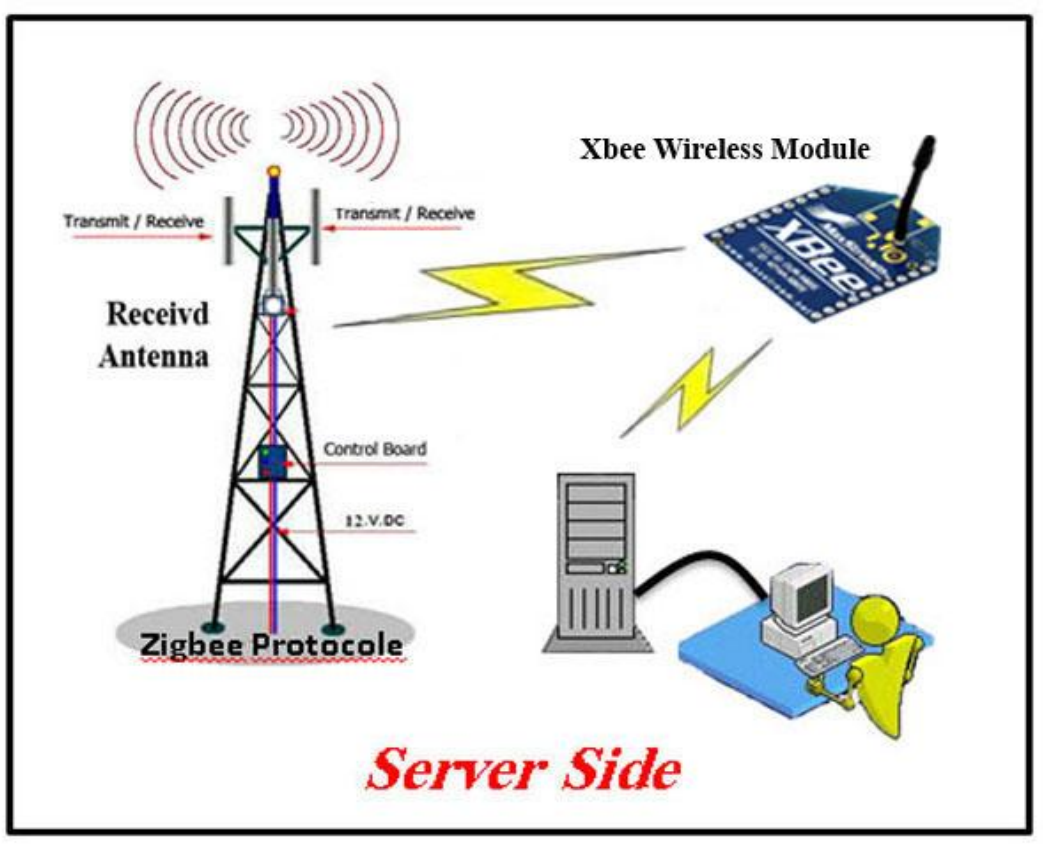

Fig. (2) Server Section based on Zigbee Protocol (Control System)

The Server part consist of personal computer to receive the eye gesture, analysis it ,matching the gesture with the database and re-send the ACTION to the driver or parking strongly by engine control[19]. Additionally GSM modem and network protocol connects with antenna both Server and Client. The purpose of this work is monitor the vehicles drivers closer when they are not with companion and feel fatigue or drowsiness or sleeping [20] .

\section{XBEE MODULE}

Xbee is small units that permit to carry out a separate-wireless connection between two Actual projects (server and client) such as carry out connection between two microcontroller units[22]. This connection technology based on Radio Frequency (RF) that have range $1 \mathrm{~km}$ or more. To assurance connection between server and client through the highway, Xbee units must be install along the highway in equal distances during zigbee protocol [23] .

\section{ZIGBEE WIRELESS SENSOR NETWORKING}

ZigBee is a wireless technology developed as an open global standard to address the unique needs of low-power wireless M2M networks. The ZigBee standard operates on the IEEE 802.15.4 physical radio specification and operates in unlicensed bands including $2.4 \mathrm{GHz}, 900 \mathrm{MHz}$ and $868 \mathrm{MHz}$ [24]. The facilities of this technology are low cost, low power, reliable, security-enabled and point to multipoint transmit/receive. ZigBee applications include Consumer Electronics, Energy Management and Efficiency, Health Care, Home Automation, driving system, Telecommunication Services, Building Automation and Industrial Automation [25].

\section{E-SAFETY OVERVIEW}

E-safety is a term for saving drivers supported by electronic processes and communication. It's utilizing information and communication technologies in a safe and responsible way. It is mainly concerned with the safeguarding of people in the digital world and making sure they feel safe when accessing new technology[21].

\section{SYSTEM BENEFITS}

The main objective of the system is to save the lives during driving for long times and ensuring driving safety. The economic benefit of the system through arrival vehicle safely and then delivery of goods to the customers safely. The useful system can be applied in many important below fields: (1) Automobiles (cars, vehicles and train) (2) Security Guard Cabins (3) Nuclear power plants, the operators must be always wakeup (4) Pilots (especially warplane , military airplane ) (5) Military application .

\section{PROPOSED SYSTEM DESCRIPTION}

The proposed system consists of two main parts, the first part is Travel Buddy (TB) installed in the vehicle and constantly monitor the driver's expressions by small static video camera installed in front of driver's face, and by GSM modem transmit these expression to the second part (server) to get the appropriate reaction. The second part is Alert Driver System (ADS), this control system turn by official control system, monitors the driver's behavior and expressions during driving and indicates to him if the driver felling drowsiness or fatigue , then the server alerts the driver through a strong voice. The system equipped with GPS system. The server side detect driver's location by GPS map during broadcasting zigbee wireless network for location tracking. Fig.(3) show a complete diagram of proposed model. The system designed to be linked to the ignition system and includes monitoring of driver's face. Additionally, the system can developed to monitor other safety features that helps to conserve the lives and safety of the drivers. Below fig.(3) show a complete infrastructure of proposed model . Any communication media consists of mainly two parts: Functional and Technical. The monitoring system (Server and Client) are composed of many important parts such as the microcontroller unit (build inside both the server and the client), set of sensors(for ACTION and REACTION) and Mesh network protocol that connect automatically with antenna that consist of two parts, first part connect directly over the Server Office, and second part connect directly with the body the vehicle via the driver's system(client). 


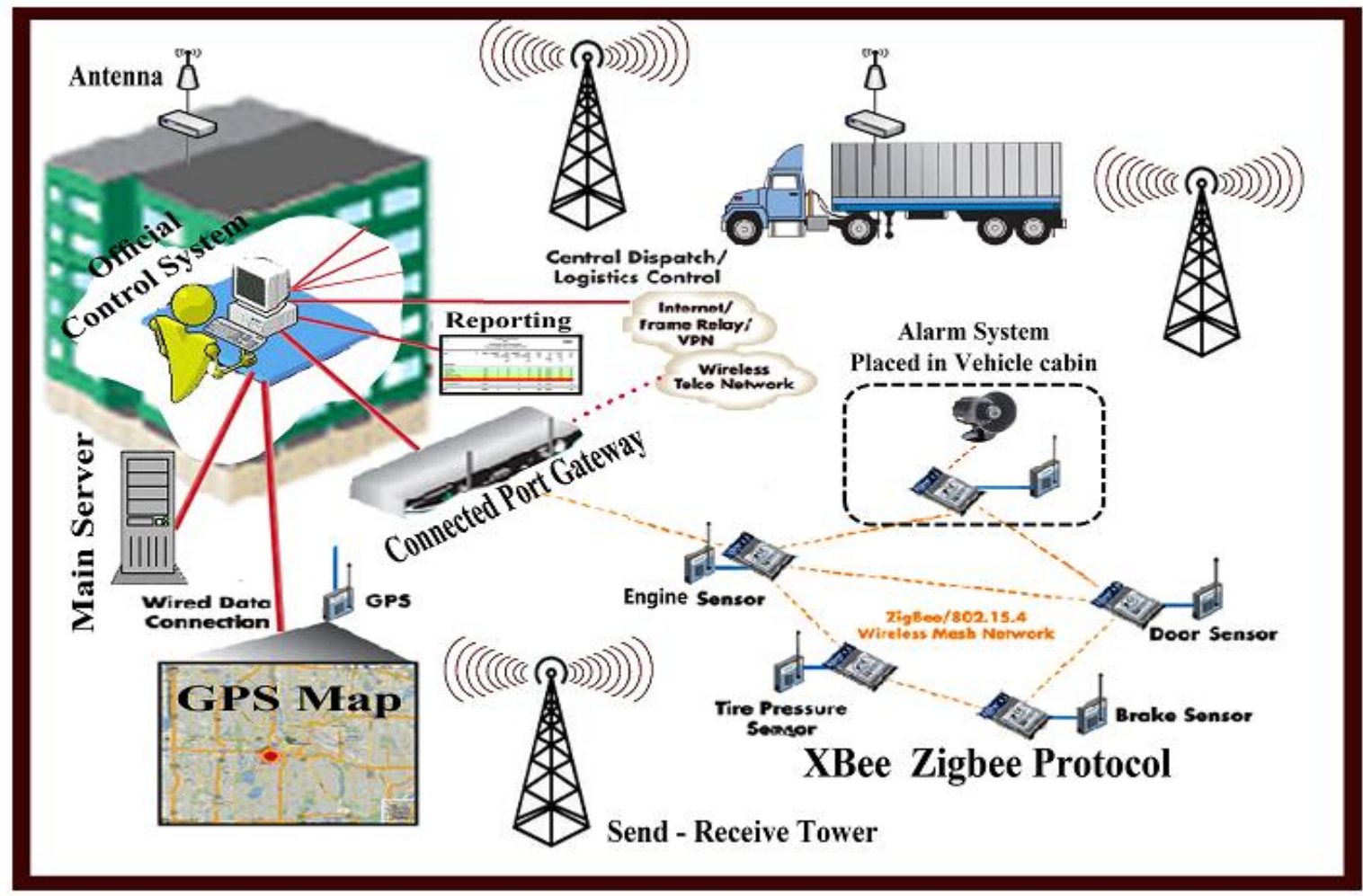

Fig. (3) A complete diagram of proposed system

The algorithm of the proposed system described briefly below:

1. Input : Eye gesture (fatigue, sleepiness, sick)

2. Output: Driver Alert, Strong Voice, No Action.

3. The procedure:

a. Signal Acquisition by CAMERA.

b. Convert acquisition signal to digital signal by computer.

c. Compare inputted signals with DATASET signals.

d. If output signal = ABNORMAL signal(fatigue, sleepiness, sick)(ACTION) then goto $f$.

e. Goto 1 f. Send ALERT to the server center (E-Safety Center) (REACTION) (STRONG VOICE) or (ENGINE CONTROL).

g. Send Alert to driver (REACTION): Continue .

h. Repeat g (REACTION) Until input signal equal to NORMAL signal (WAKE Signal) .

i. End.

\section{EXPERIMENTAL RESULT}

The experiments were carried out in two phases. In the first phase, the images by using a video camera and in the next phase, these images were used as inputs to the system to detect driver fatigue. Table (1) shows the system experimental results.

Table (1) Driver's Eye and system Statues

\begin{tabular}{|c|c|c|c|c|c|}
\hline $\begin{array}{c}\text { Eye } \\
\text { Statues }\end{array}$ & $\begin{array}{c}\text { Blinkin } \\
\text { g Rate }\end{array}$ & Accuracy & $\begin{array}{c}\text { Driver } \\
\text { Statues }\end{array}$ & Fatigue & $\begin{array}{c}\text { System } \\
\text { Reaction }\end{array}$ \\
\hline Drowsy & $93 \%$ & $88 \%$ & Abnormal & Yes & Sound Alert \\
\hline $\begin{array}{c}\text { Sleepine } \\
\text { ss }\end{array}$ & $88 \%$ & $91 \%$ & Abnormal & Yes & Sound Alert \\
\hline Sick & $79 \%$ & $86 \%$ & Abnormal & Yes & Sound Alert \\
\hline Awake & $41 \%$ & $90 \%$ & Normal & No & No Action \\
\hline
\end{tabular}

\section{CONCLUSION AND FEATURE WORKS}

In order to construct a safety driver system, this technique design by using Zigbee protocol and GSM modem due to the big facility and flexibility of this protocol and this modem for data transmitting and receiving. This technique based on analysis the driver's face expression, eyes expression and then received the compatible reaction from server. This system studying the number of consecutive frames where the eyes are closed. At that point it may be too late to issue the warning. By studying eye movement patterns, it is possible to find a method to generate the warning sooner. The driver may feel discomfort because of the sound of the alarm system would be higher where the sound begins when the eye "fall", but the big interest of this system makes the system more acceptance. 


\section{REFERENCES}

[1] Saranummi, N. In the spotlight "Health information systems". IEEE Rev. Biomed. Eng. 2008, 1, 15-17.

[2] Goldschmidt, P.G. HIT and MIS: "Implications of health information technology and medical information systems". Communication. ACM 2005, 48, 69-74.

[3] C. Jiangwei, J. Lisheng, T. Bingliang, S. Shuming, W. Rongben. 2004. "A monitoring method of driver mouth behavior based on machine vision". Proceedings of IEEE Intelligent Vehicles Symposium, Parma, Italy.

[4] S. Ribarić, J. Lovrenčić, and N. Pavešić, "A NeuralNetwork-Based System for Monitoring Driver Fatigue", in Proc. of the 15th IEEE Mediterranean Electrotechnical Conference (MELECON), pp. 1356- 1361, 2010.

[5] S. Lal, A. Craig, P. Boord, L. Kirkup, and H. Nguyen, "Development of an algorithm for an eeg-based driver fatigue countermeasure," Journal of Safety Research, vol. 1, no. 34, pp. 321-328, Febuary 2003.

[6] Q. Ji, Z. Zhu, and P. Lan, "Real-time nonintrusive monitoring and prediction of driver fatigue," IEEE transactions on vehicular technology, vol. 53, no. 4, pp. 1052-1068, July 2004

[7] T. Wang, P. Shi. (2005). "Yawning detection for determining driver drowsiness". Proceedings of IEEE Intl. Workshop VLSI Design and Video Technology, China.

[8] L.M. Bergasa, J. Nuevo, M.A. Sotalo, and M. Vazquez, "Real-time system for monitoring driver Vigilance," in Proc. Intelligent Vehicle Symp., Parma, Italy, pp.78-83, 2004.

[9] J. Nesvadba, A. Hanjalic, P. Fonseca, B. Kroon, H. Celik, E. Hendriks, 'Towards a real-time and distributed system for face detection, pose estimation and facerelated features', Invited Paper, Proc. Int. Con. on Methods and Techniques in Behavioral Research, Wageningen, The Netherlands, 2005.

[10] K.Subhashini and Y. Bahindwar, "A Dedicated System for Monitoring of Driver's Fatigue", International Journal of Innovative Research in Science, Engineering and Technology Vol. 1, Issue 2, December 2012

[11] V. Ratanavaraha and D. Watthanaklang,"Road Safety Audit: Identification of Bus Hazardous Location in Thailand", IJST Indian international journal.

[12] Aryuanto and F. Yudi Limpraptono ," A Vision Based System for monitoring driver fatigue" A vision-based system for monitoring driver fatigue" ,Yogyakarta, 14 November 2009.

[13] R. Coetzer," Driver fatigue detection based on eye tracking", Department of Electrical, Electronic and Computer Engineering. University of Pretoria, Pretoria.

[14] Qiang Ji1 and Xiaojie Yangthis ," Real-Time Eye, Gaze, and Face Pose Tracking for Monitoring Driver Vigilance", Real-Time Imaging 8, 357-377 (2002), available online at http://www.idealibrary.com.

[15] Pavan Patidar, Manoj Gupta, Sumit, Ashok, "Image Denoising By Various Filters For Different Noises", International Journal of Computer Applications (0975 8887)Volume 9-No.4, November 2010.
[16] Hawlader Abdullah Al-Mamun, Nadim Jahangir, Md. Shahedul Islam and Md. Ashraful Islam(2009), "Eye Detection in Facial Image by Genetic Algorithm Driven Deformable Template Matching", IJCSNS International Journal of Computer Science and Network Security, Vol 9, Issue 8, August 2009, Page(s): 287-294.

[17] Jie Tang, Zuhua Fang, Shifeng Hu, Ying Sun, (2010), "Driver Fatigue Detection Algorithm Based on Eye Features," IEEE proceedings of 2010 Seventh International Conference on "Fuzzy Systems and Knowledge Discovery" (FSKD 2010).

[18] Wenhui Dong, Xiuojuan Wu, (2005), "Driver Fatigue Detection Based on the Distance of Eyelid," IEEE Int Workshop on "VLSI Design \& Video Tech.", Issue May 28-30,2005, Page(s):365-368.

[19] Wen-Bing Horng, Chih-Yuan Chen, Yi Chang, Chuu-Hai Fan, (2004), "Driver Fatigue Detection Based on Eye Tracking and Dynamic Template Matching", IEEE Proceedings of International Conference on -Networking, Sensing \& Control, Taipei, Taiwan.

[20] Xiao Fan Bao-Cai Yin Yan-Feng Sun "Yawning Detection for Monitoring Driver Fatigue" IEEE Machine Learning and Cybernetics, 2007 International Conference on 19-22 Aug. 2007.

[21] M. Divjak and H. Bischof, "Eye blink based fatigue detection for prevention of computer vision syndrome," in the IAPR Conference on Machine Vision Applications, 2009, pp. 350-353.

[22] Tamilselvan, G.M. and A. Shanmugam. Multi hopping effect of Zigbee nodes coexisting with WLAN nodes in heterogeneous network environment in Cognitive Wireless Systems (UKIWCWS), 2009 First UK-India International Workshop on. 2009.

[23] Dissanayake, S.D., et al. Zigbee Wireless Vehicular Identification and Authentication System. in Information and Automation for Sustainability, 2008. ICIAFS 2008 4th International Conference on. 2008.

[24] Zeghdoud, M., P. Cordier, and M. Terre. Impact of Clear Channel Assessment Mode on the Performance of ZigBee Operating in a WiFi Environment. in OperatorAssisted (Wireless Mesh) Community Networks, 2006 1st Workshop on. 2006.

[25] Dissanayake, S.D., et al. "Zigbee Wireless Vehicular Identification and Authentication System in Information and Automation for Sustainability", 2008. ICIAFS 2008. 4th International Conference on. 2008.

\section{AUTHOR'S PROFILE}

M.Sc. Azmi Shawkat Abdulbaki obtained his M.Sc. in Computer Science from University of Anbar. He is currently instructor of Computer Science in College of Computer ,University of Anbar ,IRAQ. His research interests are Artificial Neural Networks, Computer Networks, Image Processing, Software Engineering, Pattern Recognition and Genetic Algorithm .

M.Sc Samir Abdulrasoul Khadim, obtained his M.Sc. in Computer Science from Okranya . He is currently instructor of Computer Science, University of Alma'arif College ,IRAQ. His research interests are Artificial Neural Networks, Computer Networks, Image Processing , Software Engineering, Pattern Recognition and Genetic Algorithm. 